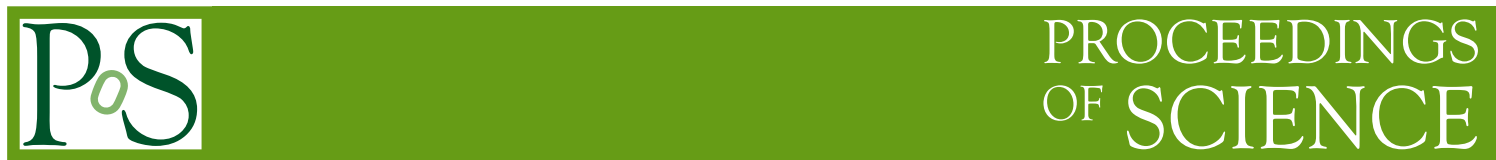

\title{
Measurement of top quark polarization with the D0 detector
}

\section{Kamil Augsten* for the D0 Collaboration}

Czech Technical University in Prague

E-mail: augsten@fnal.gov

We present a measurement of the top quark polarization in the $t \bar{t}$ pair production in $p \bar{p}$ collisions at the Fermilab Tevatron collider at $\sqrt{s}=1.96 \mathrm{TeV}$. We use the full Run II data sample corresponding to $9.7 \mathrm{fb}^{-1}$ of integrated luminosity recorded with the D0 detector. We consider the final state containing a lepton (electron or muon) and at least three jets. The polarization is measured through the distribution of lepton angles. We consider three different axes for the polarization measurement: the beam axis, the helicity axis, and the axis normal to the $t \bar{t}$ production plane. This is the first measurement of the transverse polarization of the top quark at a hadron collider. The observed top quark polarizations for each of the three axes are consistent with standard model predictions of nearly zero polarization.

8th International Workshop on Top Quark Physics, TOP2015

14-18 September, 2015

Ischia, Italy

${ }^{*}$ Speaker. 
The standard model (SM) predicts that top quark pairs are produced almost unpolarized at the Tevatron (a small longitudinal polarization is generated by SM parity-violating weak interactions [1]), while various models beyond the SM predict non-zero polarization of the top quark pairs. The transverse polarization is allowed in strong interaction processes. The SM predictions are shown in Table 3. The top quark polarization $P_{\hat{n}}$ can be measured in the top quark rest frame through the angular distribution of the top quark decay products with respect to a chosen axis $\hat{n}[1,2]$ : $\frac{1}{\Gamma} \frac{d \Gamma}{d \cos \theta_{i, \hat{n}}}=\frac{1}{2}\left(1+P_{\hat{n}} \kappa_{i} \cos \theta_{i, \hat{n}}\right)$, where $i$ is the decay product, $\kappa_{i}$ its spin analyzing power $(\approx 1$ for $\ell, 0.97$ for $d$-type quark, -0.4 for $b$-quark, and -0.3 for neutrino and $u$-type quark $[2,3])$, and $\theta_{i, \hat{n}}$ is the angle between the direction of the decay product $i$ and the quantization axis $\hat{n}$. The spin quantization axis choices are: the beam axis $\hat{b}$, given by the direction of the proton beam, the helicity axis $\hat{h}$, given by the direction of the parent top quark, and the transverse axis $\hat{t}$, given as perpendicular to the production plane defined by the proton and parent top quark directions $[4,5]$.

This analysis is performed using the lepton + jets final state from data collected by the D0 detector [6]. We require one isolated electron [7] or muon [8] with transverse momentum $p_{T}>$ $20 \mathrm{GeV}$ and $|\eta|<1.1$ or $|\eta|<2$, missing transverse momentum $\not p_{\mathrm{T}}>20 \mathrm{GeV}$, at least three jets with $p_{T}>20 \mathrm{GeV}$ within $|\eta|<2.5$, and $p_{T}>40 \mathrm{GeV}$ for the jet of highest $p_{T}$. At least one jet per event is required to be identified as originating from a $b$ quark ( $b$-tagged) through the use of a multivariate algorithm [9]. Additional selection requirements are applied same way as described in Ref. [10]. In addition, we also require that the curvature of the track associated with the lepton is well measured to reduce lepton charge misidentification.

We use Monte Carlo simulated $t \bar{t}$ events generated using the MC@ NLO event generator version 3.4 [11] or the ALPGEN event generator version 2.11 [12]. Parton showering, hadronization, and underlying event modeling are performed with HERWIG [13] for MC@ NLO events and with PYTHIA [14] for ALPGEN events. The detector is simulated using GEANT3 [15]. The background samples are used same as defined in Ref. [10].

A $\chi^{2}$-based constrained kinematic fit is utilized to associate the observed leptons and jets with the individual top quarks as described in [16]. The kinematic fit algorithm includes a technique that allows reconstruction of events with lepton and three jets [17]. All possible combinations of objects are considered and weighted by the $\chi^{2}$ of the kinematic fit solution.

To determine the sample composition we choose input variables that form a kinematic discriminant based on the approximate likelihood ratio between the $t \bar{t}$ and $W+$ jets predictions. The input variables are selected for good separation between the $t \bar{t}$ events and the $W+$ jets events and are required to be well modeled and not strongly correlated with one another or to the lepton polar angles. Such variables are $k_{T}^{\text {min }}$,

\begin{tabular}{lcccc}
\hline \hline & \multicolumn{2}{c}{3 jets } & \multicolumn{2}{c}{$\geq 4$ jets } \\
Source & $e+$ jets & $\mu+$ jets & $e+$ jets & $\mu+$ jets \\
\hline$W+$ jets & $1741 \pm 26$ & $1567 \pm 15$ & $339 \pm 3$ & $295 \pm 3$ \\
Multijet & $494 \pm 7$ & $128 \pm 3$ & $147 \pm 4$ & $49 \pm 2$ \\
Other Bg & $446 \pm 5$ & $378 \pm 2$ & $87 \pm 1$ & $73 \pm 1$ \\
$t \bar{t}$ signal & $1200 \pm 25$ & $817 \pm 20$ & $1137 \pm 24$ & $904 \pm 23$ \\
\hline Sum & $3881 \pm 37$ & $2890 \pm 25$ & $1710 \pm 25$ & $1321 \pm 23$ \\
\hline Data & 3872 & 2901 & 1719 & 1352 \\
\hline \hline
\end{tabular}

Table 1: Estimated number of events after selection and after determination of the sample composition from maximum-likelihood fit to the discriminant distribution. The quoted uncertainties are statistical. 
aplanarity, $H_{T}^{\ell}$ and others described in [18]. The $\ell+3$ jet and $\ell+\geq 4$ jet samples are each divided into 3 sub-channels according to the number of $b$-tagged jets: $0,1, \geq 2$ for the sample composition fit. The zero- $b$-tag channels are used only to determine the sample composition and background calibration, not to measure the polarization. The sample composition is determined from a simultaneous maximum-likelihood fit to the discriminant distribution for the $t \bar{t}$ signal and $W+$ jets background and the resulting selection is summarized in Table 1.

We need the lepton angular distributions for the $W+$ jets events to be properly modeled, so it does not bias the final template fit, which is especially important in $\ell+3$ jet sample. To obtain a good model of the $W+$ jets templates, we use the data in the $\ell+3$ jets and $0 b$-tag control sample, which is dominated by the $W+$ jets background with more than $70 \%$ contribution. The $t \bar{t}$ events and other background components are subtracted from data. We reweight the $W+$ jets $\mathrm{MC}$ events so that the $\cos \theta_{\ell, \hat{n}}$ distribution shows good agreement with the data. The correction obtained in the control sample is propagated to the other samples.

\begin{tabular}{lccc}
\hline \hline Source & Beam & Helicity & Transverse \\
\hline Signal and background modeling & \pm 0.019 & \pm 0.022 & \pm 0.009 \\
Detector modeling & \pm 0.017 & \pm 0.034 & \pm 0.011 \\
Method & \pm 0.014 & \pm 0.008 & \pm 0.005 \\
\hline Total systematic uncertainty & \pm 0.030 & \pm 0.041 & \pm 0.015 \\
Total statistical uncertainty & \pm 0.046 & \pm 0.044 & \pm 0.030 \\
Total uncertainty & \pm 0.055 & \pm 0.060 & \pm 0.034 \\
\hline \hline
\end{tabular}

Table 2: Summary of uncertainties on the measured top quark polarization along three axes.

To measure polarization, a template fit to the reconstructed lepton $\cos \theta_{\hat{n}}$ distribution is made. The $t \bar{t}$ MC sample is only generated with zero polarization. We then obtain two templates of +1 and -1 polarization by reweighting the simulated $t \bar{t}$ events based on the double differential distribution:

$$
\frac{1}{\Gamma} \frac{d \Gamma}{d \cos \theta_{1} \cos \theta_{2}}=\frac{1}{4}\left(1+\kappa_{1} P_{\hat{n}, 1} \cos \theta_{1}+\rho \kappa_{2} P_{\hat{n}, 2} \cos \theta_{2}-\kappa_{1} \kappa_{2} C \cos \theta_{1} \cos \theta_{2}\right),
$$

where index 1 represents the $t$ decay product ( $\ell$ or $d$-type quark) and index 2 the $\bar{t}$ decay product, $C$ stands for the anti-correlation factor, representing the spin correlation of the $t \bar{t}$ pair. We use the SM values $C=-0.368$ (helicity axis) and $C=0.791$ (beam axis), both calculated at NLO in QCD and electroweak couplings [1]. The spin correlation factor is not known for the transverse axis and we set $C=0$. In the SM with $C P$ invariance, the relative sign factor $\rho$ takes the value +1 for the helicity basis and -1 for the beam and transverse bases $[1,19]$. A simultaneous fit is performed to the data using the $P=+1$ and -1 signal templates and the background template normalized to the expected background contribution. The observed polarization is taken as $P=f_{+}-f_{-}$, where $f_{ \pm}$are the fractions of $P=+1$ and -1 returned in the fit. The distributions of the $\cos \theta_{\ell, \hat{n}}$ from $t \bar{t}$ decay are shown in Fig. 1.

A simultaneous measurement of the top quark polarization and the forward-backward asymmetry in the dilepton final states [20] observed a correlation between the two measurements. This correlation is due to acceptance and resolution effects in the event kinematic reconstruction. We determine the dependence of the observed polarization on the parton level forward-backward 
asymmetry, $A_{F B}$ and we perform a correction for the difference between the nominal MC@NLO production-level and the actual NNLO calculation [21].
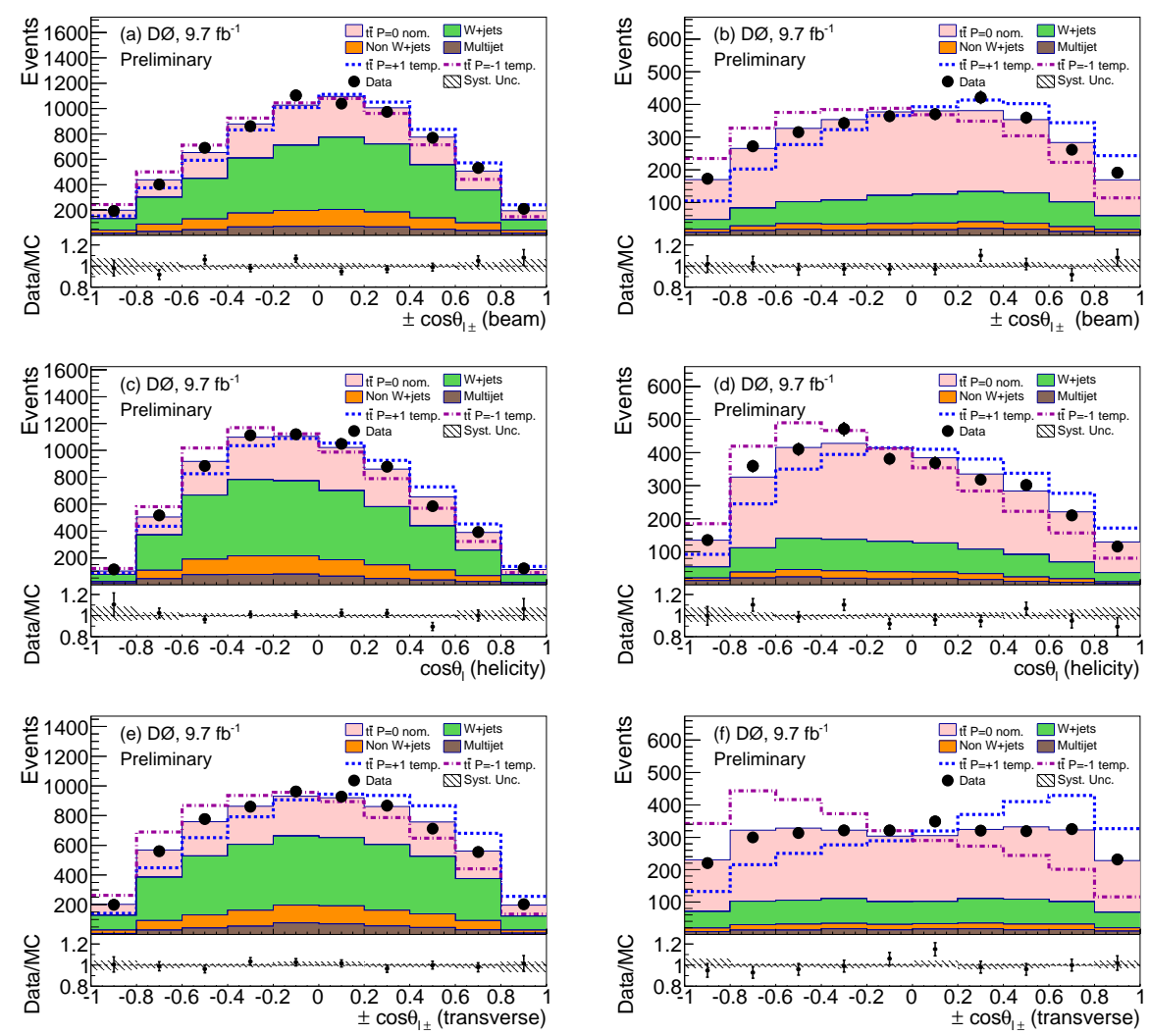

Figure 1: The combined $e+$ jets and $\mu+$ jets $\cos \theta$ distributions for data, expected backgrounds and signal templates for $P=-1,0$ and +1 . Panels (a), (c), and (e) represent selection with exactly three jets. (b), (d), and (f) represent selection with four or more jets. The hashed area represents systematic uncertainty.

Three categories of systematic uncertainties are evaluated using fully simulated events including background contributions: modeling of signal and background events, uncertainties in the simulation of the detector response, and uncertainties in procedures used and assumptions made in the analysis as shown in Table 2. More details about the systematic uncertainties and

\begin{tabular}{lcc}
\hline \hline Axis & Measured polarization $P_{\hat{n}}$ & SM prediction \\
\hline Beam & $+0.070 \pm 0.055$ & $-0.002[23]$ \\
Helicity & $-0.102 \pm 0.060$ & $-0.004[23]$ \\
Transverse & $+0.040 \pm 0.034$ & $+0.011[5]$ \\
\hline \hline
\end{tabular}

Table 3: Measured top quark polarizations and the respective SM predictions. determination can be found in $[18,22]$.

The final measured polarizations for the three spin quantization bases are shown in Table 3 . The results are consistent with zero and with the predicted SM values. The polarization along the transverse axis has been measured for the first time at a hadron collider and the longitudinal polarization results are the most precise results based on $p \bar{p}$ collisions. 


\section{References}

[1] W. Bernreuther and Z. -G. Si, "Distributions and correlations for top quark pair production and decay at the Tevatron and LHC.," Nucl. Phys. B 837 (2010) 90.

[2] D. Krohn, T. Liu, J. Shelton and L. -T. Wang, "A Polarized View of the Top Asymmetry," Phys. Rev. D 84 (2011) 074034.

[3] A. Brandenburg, Z. G. Si and P. Uwer, "QCD corrected spin analyzing power of jets in decays of polarized top quarks,” Phys. Lett. B 539 (2002) 235.

[4] W. Bernreuther, A. Brandenburg and P. Uwer, "Transverse polarization of top quark pairs at the Tevatron and the large hadron collider," Phys. Lett. B 368 (1996) 153.

[5] M. Baumgart and B. Tweedie, "Transverse Top Quark Polarization and the $t \bar{t}$ Forward-Backward Asymmetry," J. High Energy Phys. 1308 (2013) 072.

[6] V. M. Abazov et al. (D0 Collaboration), “The Upgraded D0 Detector," Nucl. Instrum. Methods Phys. Res. Sec. A 565, 463 (2006).

[7] V. M. Abazov et al. (D0 Collaboration), "Electron and Photon Identification in the D0 Experiment," Nucl. Instrum. Meth. Phys. Res. Sec A 750, 78 (2014).

[8] V. M. Abazov et al. (D0 Collaboration), "Muon reconstruction and identification with the Run II D0 detector," Nucl. Instrum. Methods Phys. Res. Sec. A 737, 281 (2014).

[9] V. M. Abazov et al. (D0 Collaboration), "Improved $b$ quark jet identification at the D0 experiment," Nucl. Instrum. Methods Phys. Res. Sec. A 763, 290 (2014).

[10] V. M. Abazov et al. (D0 Collaboration), "Measurement of differential $t \bar{t}$ production cross sections in $p \bar{p}$ collisions," Phys. Rev. D 90, 092006 (2014).

[11] S. Frixione and B. R. Webber, "Matching NLO QCD computations and parton shower simulations," JHEP 0206 (2002) 029; S. Frixione et al., "Matching NLO QCD and parton showers in heavy flavour production," JHEP 0308 (2003) 007.

[12] M. L. Mangano, M. Moretti, F. Piccinini, R. Pittau and A. D. Polosa, "ALPGEN, a generator for hard multiparton processes in hadronic collisions," JHEP 0307, 001 (2003).

[13] G. Corcella et al., "HERWIG 6: An Event generator for hadron emission reactions with interfering gluons (including supersymmetric processes)," JHEP 0101, 010 (2001).

[14] T. Sjøstrand, S. Mrenna, and P. Skands, “PYTHIA 6.4 physics and manual,” J. High Energy Phys. 05 (2006) 026.

[15] R. Brun and F. Carminati, CERN Program Library Long Writeup W5013 (1993) (unpublished).

[16] V. M. Abazov et al. (D0 Collaboration), "Measurement of the forward-backward asymmetry in top quark-antiquark production in $p \bar{p}$ collisions using the lepton+jets channel," Phys. Rev. D 90, 072011 (2014).

[17] R. Demina, A. Harel and D. Orbaker, "Reconstructing $t \bar{t}$ events with one lost jet," Nucl. Instrum. Methods Phys. Res. Sec. A 788, 128 (2015).

[18] The D0 Collaboration, D0 Note 6471-CONF.

[19] W. Bernreuther, “Top quark physics at the LHC,” J. Phys. G 35, 083001 (2008). 
[20] V. M. Abazov et al. (D0 Collaboration), "Simultaneous Measurement of Forward-Backward Asymmetry and Top Polarization in Dilepton Final States from $t \bar{t}$ Production at the Tevatron," Phys. Rev. D 92, 052007 (2015).

[21] M. Czakon, P. Fiedler and A. Mitov, "Resolving the Tevatron top quark forward-backward asymmetry puzzle," arXiv:1411.3007 [hep-ph].

[22] V. M. Abazov et al. (D0 Collaboration), "Measurement of the forward-backward asymmetry in the distribution of leptons in $t \bar{t}$ events in the lepton+jets channel," Phys. Rev. D 90, 072001 (2014).

[23] W. Bernreuther, M. Fucker and Z. G. Si, "Weak interaction corrections to hadronic top quark pair production: Contributions from quark-gluon and b anti-b induced reactions," Phys. Rev. D 78, 017503 (2008); W. Bernreuther and Z. -G. Si, unpublished e-mail communication. 